\title{
Characterization of a rationally engineered phaCAB operon with a hybrid promoter design
}

lain Bower ${ }^{\# 1,3}$, Bobby Wenqiang Chi ${ }^{\# 1,2}$, Matthew Ho Wai Chin ${ }^{\# 1,2}$, Sisi Fan $^{\# 1,3}$, Margarita Kopniczky ${ }^{\# 1,3}$, Jemma Pilcher ${ }^{\# 1,3}$, James Strutt $^{\# 1,3}$, Richard Kelwick ${ }^{1,3^{*}}$, Alexander Webb ${ }^{1,3}$, Kirsten Jensen ${ }^{1,3}$, Guy-Bart Stan ${ }^{1,2}$, Richard Kitney ${ }^{1,2^{*}}$ and Paul Freemont ${ }^{1,3^{*}}$

\section{Author affiliations:}

${ }^{1}$ Centre for Synthetic Biology and Innovation,

${ }^{2}$ Department of Bioengineering, ${ }^{3}$ Department of Life Sciences, Imperial College London, South Kensington Campus, London SW7 2AZ, UK

\section{Author Contributions}

\# authors are equal first author, each of whom were equally involved in the design of the iGEM project and the molecular and microbial work carried out. R. K., A. W., K. J., GB. S., R. K., and P. $\mathrm{F}$. supervised and coordinated the study.

* to whom correspondence should be addressed

Paul Freemont p.freemont@imperial.ac.uk

Richard Kitney r.kitney@imperial.ac.uk

Richard Kelwick r.kelwick@imperial.ac.uk

\section{Conflict of interest:}

The authors declare no conflict of interest and no competing financial interests. 


\begin{abstract}
:
Biopolymers, such as poly-3-hydroxy-butyrate $(\mathrm{P}(3 \mathrm{HB}))$ are produced as a carbon store in an array of organisms and exhibit characteristics which are similar to oil-derived plastics, yet have the added advantages of biodegradability and biocompatibility. Despite these advantages, $\mathrm{P}(3 \mathrm{HB})$ production is currently more expensive than the production of oil-derived plastics, and therefore more efficient $\mathrm{P}(3 \mathrm{HB})$ production processes are required. In this study, we describe the model-guided design and experimental characterization of several engineered $\mathrm{P}(3 \mathrm{HB})$ producing operons. In particular, we describe the characterization of a novel hybrid phaCAB operon that consists of a dual promoter (native and $\mathrm{J} 23104$ ) and RBS (native and B0034) design. $\mathrm{P}(3 \mathrm{HB})$ production was around six-fold higher in hybrid phaCAB engineered Escherichia coli in comparison to $E$. coli engineered with the native phaCAB operon from Ralstonia eutropha $\mathrm{H} 16$. The hybrid phaCAB operon represents a step towards the more efficient production of $\mathrm{P}(3 \mathrm{HB})$, which has an array of applications from 3D printing to tissue engineering.
\end{abstract}

Key Words: Poly-3-hydroxy-butyrate; hybrid promoter; iGEM

\title{
Introduction:
}

Conventional oil-derived polyolefin plastics exhibit useful characteristics that have extensive commercial applications. However, the accumulation of plastics in the environment and the non-renewable source of polyolefin plastics have stimulated interest in sustainable sources of plastic production. Biopolymers, such as poly-3-hydroxy-butyrate $(\mathrm{P}(3 \mathrm{HB}))$ are produced as a carbon store in an array of organisms and exhibit characteristics which are similar to oil-derived plastics ${ }^{(1,2)}$. Furthermore, $\mathrm{P}(3 \mathrm{HB})$ has the added advantages of biodegradability, biocompatibility and, based upon several life cycle analyses, $\mathrm{P}(3 \mathrm{HB})$ production is more environmentally sustainable than polyolefin plastic production $^{(1,3,4)}$. Despite these advantages, $\mathrm{P}(3 \mathrm{HB})$ production is currently more expensive than the production of oil-derived plastics, and therefore more efficient $\mathrm{P}(3 \mathrm{HB})$ production processes are required ${ }^{(5)}$. Genetic engineering approaches in which the $\mathrm{P}(3 \mathrm{HB})$-producing operon, phaCAB, is cloned into Escherichia coli, have pioneered the industrial production of $\mathrm{P}(3 \mathrm{HB})^{(6)}$. More recently, synthetic biology approaches involving the rational 
engineering of the phaCAB operon ${ }^{(7)}$, and metabolic engineering strategies $^{(8)}$ have continued to increase the efficiency of $P(3 \mathrm{HB})$ production. 'Team Plasticity', comprising undergraduate students from Imperial College London, set out to engineer a more efficient phaCAB operon as part of a project in the 2013 International Genetically Engineered Machine Competition (iGEM). The broader project aim was to exploit non-recyclable waste as a carbon source that could be utilized by engineered $E$. coli tasked with the de novo synthesis of $\mathrm{P}(3 \mathrm{HB})$. In this paper we report on the characterization of a novel hybrid promoter phaCAB operon, which was generated during the project.

\section{Results and Discussion:}

The phaCAB operon from Ralstonia eutropha $\mathrm{H} 16$, is the most extensively studied $\mathrm{P}(3 \mathrm{HB})$ synthesis operon ${ }^{(2)}$. It consists of three enzymes, which through a multi-stage enzymatic process generate $\mathrm{P}(3 \mathrm{HB})$ inside the cell from the central metabolite acetyl-CoA ${ }^{(1)}$. The process is shown in Figure $1 \mathrm{~A}$ and is briefly summarised here. Firstly, PhaA (3-ketothiolase) combines two molecules of acetylCoA to form acetoacetyl-CoA. Next, PhaB (acetoacetyl-CoA reductase) reduces acetoacetyl-CoA to form (R)-3-hydroxybutylCoA, which is then polymerised by PhaC (PHA synthase) to form poly-3-hydroxy-butyrate $\mathrm{P}(3 \mathrm{HB})$. The phaCAB operon from $R$. eutropha $\mathrm{H} 16$ was originally cloned into $E$. coli in the late $1980 \mathrm{~s}^{(2)}$. More recently the Tokyo Tech 2012 iGEM team (http://2012.igem.org/Team:Tokyo_Tech) cloned, characterised and standardized the native phaCAB operon into a biobrickcompatible format for the synthetic biology community.

In order to increase $\mathrm{P}(3 \mathrm{HB})$ production, several novel phaCAB operons were engineered during the course of our project (Figure 1B). The constitutive phaCAB operon (BBa_K1149052) was designed such that the native promoter and RBS were replaced with a strong Anderson promoter (J23104) and the RBS B0034. Design considerations were based upon modeling simulations of the PhaCAB pathway in engineered E. coli. A sensitivity analysis of the synthetic pathway revealed that increasing expression of phaB would increase $\mathrm{P}(3 \mathrm{HB})$ production. In order to increase expression of phaB, further simulations predicted that of the several designs that were tested, it was the constitutive operon design that would result in an increase in $\mathrm{P}(3 \mathrm{HB})$ production (Figure 2). The hybrid operon design (BBa_K1149051) was constructed in parallel to the constitutive operon and is noted for its 
dual promoter and RBS combinations (Figure 1B).

E. coli MG1655 carrying either the empty vector (BBa_K608002), native phaCAB (BBa_K934001), constitutive phaCAB (BBa_K1149052) or hybrid phaCAB (BBa_K1149051) were cultured as described in Methods. $\mathrm{P}(3 \mathrm{HB})$ was purified using sodium hypochlorite. Purified $\mathrm{P}(3 \mathrm{HB})$ from each of the engineered populations were weighed and compared (Figure 3). Average $\mathrm{P}(3 \mathrm{HB})$ production was around two-fold higher in constitutive phaCAB-engineered E. coli $(0.49 \mathrm{~g} / \mathrm{L}$ S.D. \pm 0.06$)$ compared to native phaCAB $(0.22 \mathrm{~g} / \mathrm{L}$ S.D. \pm 0.18$)$, while average $\mathrm{P}(3 \mathrm{HB})$ production was six-fold higher in hybrid phaCAB-engineered $E$. coli (1.47 g/L S.D. \pm 0.48$)$, and empty vector-engineered E. coli did not produce detectable levels of $\mathrm{P}(3 \mathrm{HB})$. Fluorescent microscopy and Nile Red staining further confirmed increased $\mathrm{P}(3 \mathrm{HB})$ production. Full details are available on the iGEM Wiki, (http://2013.igem.org/Team:Imperial_College) .

It is likely that the dual promoter and RBS design (Figure 1B) of the hybrid system results in a higher level of mRNA transcript production and/or ribosome recruitment and thus an increase in the expression of the PhaCAB enzymes. Yet interestingly, $\mathrm{P}(3 \mathrm{HB})$ production from the hybrid operon was greater than the combined $\mathrm{P}(3 \mathrm{HB})$ production of the native and constitutive operons. Together, these data suggest that the hybrid promoter performs a multiplicative, rather than an additive combination of the native and constitutive promoter designs. Unlocking the design rules of the hybrid promoter may have applicability that extends beyond the phaCAB operon. Additionally, model-guided optimisation of the PhaCAB pathway led to the generation of the constitutive operon design, which in combination with the hybrid promoter, represent an emerging family of novel, rationally engineered $\mathrm{P}(3 \mathrm{HB})$ producing operons. These engineered operons in combination with additional synthetic biology approaches ${ }^{(7,8)}$ and the switch towards low-cost carbon sources, such as mixed, non-recyclable waste, will further increase the efficiency and commercial viability of $\mathrm{P}(3 \mathrm{HB})$ production. 

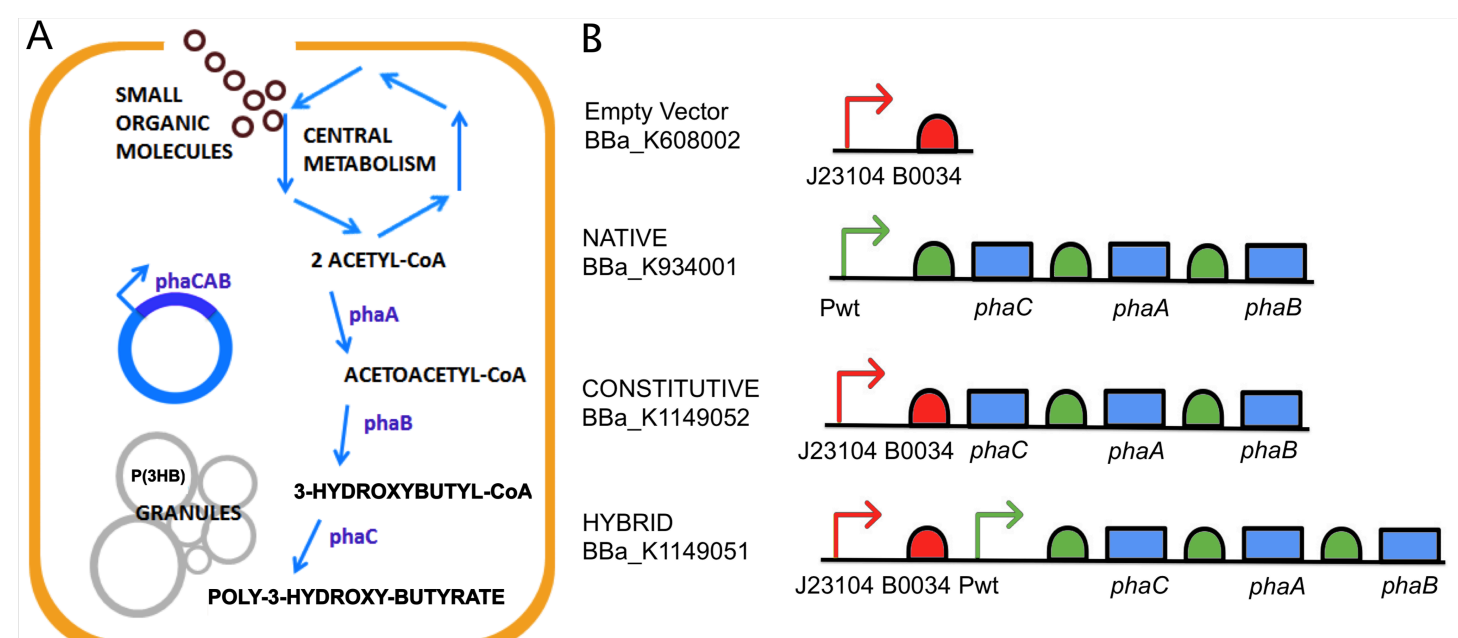

Figure 1. phaCAB pathway and constructs

(A) Schematic of poly-3-hydroxy-butyrate $(\mathrm{P}(3 \mathrm{HB}))$ production via the phaCAB operon pathway. (B) The constructs utilized in this study. Abbreviations: Pwt (wildtype promoter; green arrow), J23104 (Anderson constitutive promoter, BBa_J23104; red arrow), B0034 (ribosomal binding site, BBa_B0034; red half-circle), phaC (PHA synthase), phaA (3-ketothiolase) and phaB (acetoacetyl-CoA reductase). Green half-circles denote native ribosomal binding sites. Construct symbols are based on the Synthetic Biology Open Language Visual (SBOLv) v1.0.0 guidelines.

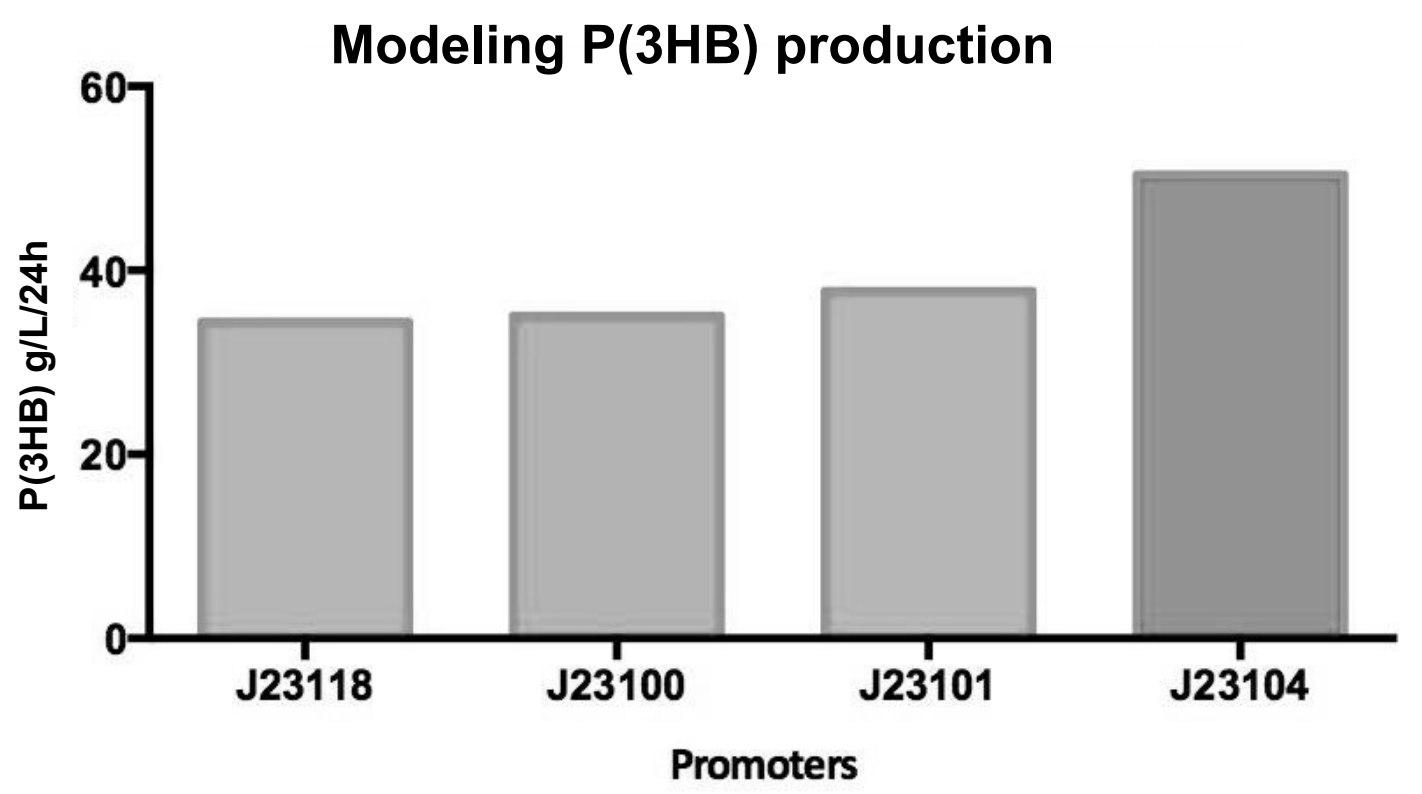

Figure 2. Modeling $\mathrm{P}(3 \mathrm{HB})$ production

Simulation data showing $\mathrm{P}(3 \mathrm{HB})$ production from different operon designs, where phaCAB expression is under the control of the indicated Anderson constitutive promoters. 


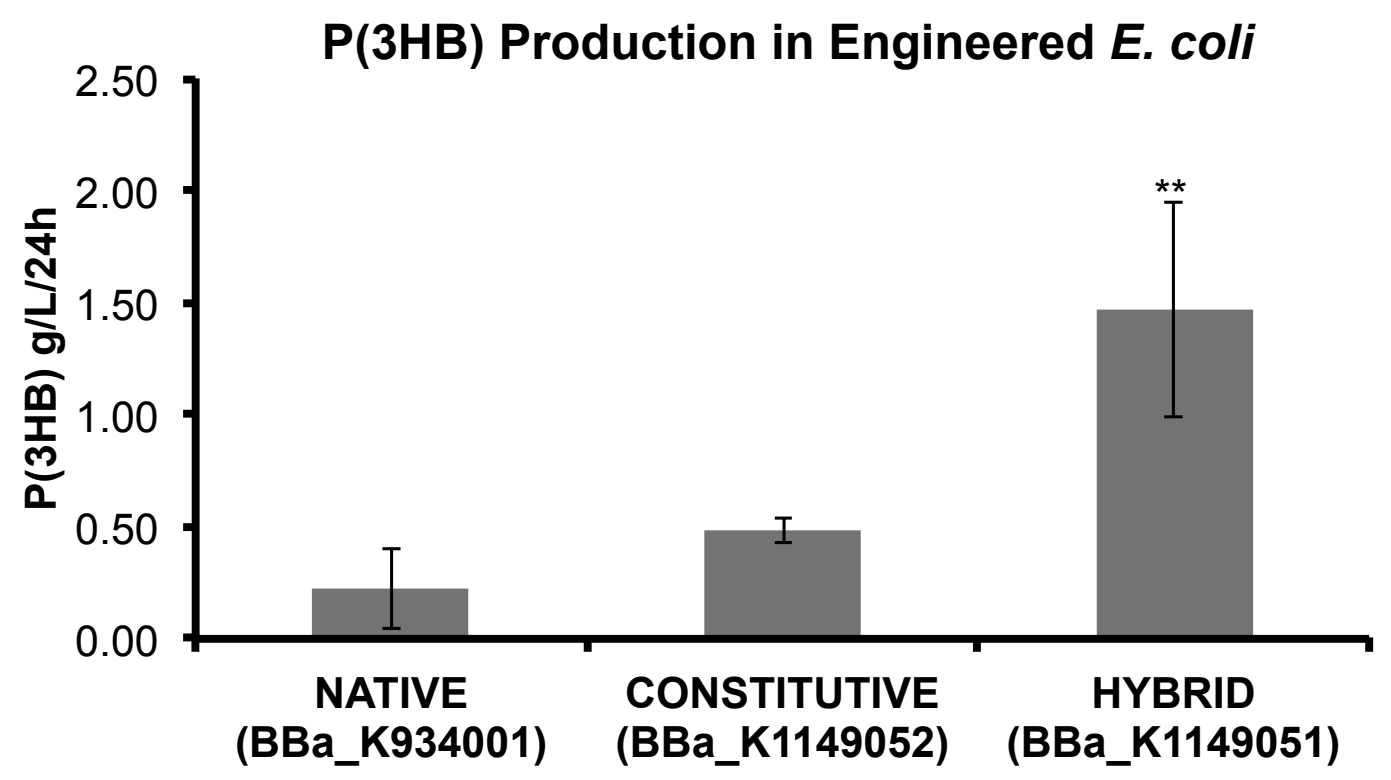

Figure 3. $\mathrm{P}(3 \mathrm{HB})$ production in $E$. coli harboring phaCAB operon constructs

E. coli MG1655 transformed with either native (BBa_K934001), constitutive (BBa_K1149052) or hybrid (BBa_K1149051) phaCAB constructs were cultured in 1 liter LB media with $3 \%$ glucose $(\mathrm{w} / \mathrm{v})$ for 24 hours. $\mathrm{P}(3 \mathrm{HB})$ was purified and weighed as described in Methods. Data represent the mean +/- the SD of three independent experiments. ${ }^{* *}$ Student t-test, $\mathrm{P}<.01$ 


\section{Methods:}

\section{Construct Assembly}

Empty vector (BBa_K608002), and native phaCAB (BBa_K934001) constructs were sourced from the 2013 distribution of the iGEM Registry of Standard Biological Parts (partsregistry.org). Constitutive phaCAB (BBa_K1149052) was generated via $\mathrm{PCR}$, with the native phaCAB operon (BBa_K934001) as the template. Primers Pha Fw 5'cgcttctagagatggctactgggaaaggagccg-3' and BBa_G1005 5'gtttcttcctgcagcggccgctactagta-3', were utilised to generate a PCR product containing the phaCAB operon but excluding the native promoter and RBS. The PCR product was cloned into the destination vector (BBa_K608002) to generate the final constitutive phaCAB operon construct. The hybrid phaCAB (BBa_K1149051) construct was generated in parallel to the constitutive phaCAB operon. The entire native phaCAB operon including the native promoter and RBS was cloned into the destination vector (BBa_K608002) to generate the final hybrid phaCAB operon construct. All constructs were generated using the IGEM submission backbone, pSB1C3 and all restriction digests utilized the standard iGEM prefix and suffix restriction sites.

\section{$\mathrm{P}(3 \mathrm{HB})$ production}

E. coli strain MG1655 carrying either empty vector (BBa_K608002), native phaCAB (BBa_K934001), constitutive phaCAB (BBa_K1149052) or hybrid phaCAB (BBa_K1149051) constructs were grown in Luria Broth (LB) media supplemented with $34 \mu \mathrm{g} / \mathrm{mL}$ Chloramphenicol (final concentration) for maintenance of plasmids. Cultures were grown overnight at $37^{\circ} \mathrm{C}$ and $200 \mathrm{rpm}$ shaking. Overnight cultures were diluted (1:200) into flasks containing 1 liter of LB, supplemented with $3 \%$ glucose $(\mathrm{w} / \mathrm{v})$ and $34 \mu \mathrm{g} / \mathrm{mL}$ Chloramphenicol. Cultures were subsequently grown for $24 \mathrm{~h}$ at $37^{\circ} \mathrm{C}$ and $200 \mathrm{rpm}$ shaking, during which $\mathrm{P}(3 \mathrm{HB})$ could be produced by phaCAB expressing $\mathrm{E}$. coli.

\section{$\mathrm{P}(3 \mathrm{HB})$ purification}

1 liter production cultures were centrifuged at $4000 \mathrm{rpm}$ for 15 minutes. Bacterial pellets were washed with phosphate-buffered saline (PBS) and then incubated with 1\% Triton-X 100 in PBS (v/v) for 30 minutes at room temperature in order to lyse the cells. Final purification of $\mathrm{P}(3 \mathrm{HB})$ utilized the sodium hypochlorite method as described in ${ }^{(9)}$. 


\section{Modelling}

The $\mathrm{P}(3 \mathrm{HB})$ synthesis model was constructed and simulated using the Simbiology toolbox of Matlab. Full details are available on the iGEM Wiki (http://2013.igem.org/Team:Imperial_College)

\section{Acknowledgements:}

We would like to thank the members of the Centre for Synthetic Biology and Innovation (CSynBI), the companies Eppendorf, Bioline, GeneArt and additionally ERASysBio for sponsoring the 2013 Imperial College London iGEM project. We would also like to thank the iGEM Foundation and its volunteers for organizing the competition that instigated this work.

\section{References:}

1. Verlinden, R. A., Hill, D. J., Kenward, M. A., Williams, C. D., and Radecka, I. (2007) Bacterial synthesis of biodegradable polyhydroxyalkanoates, Journal of applied microbiology 102, 1437-1449.

2. Sudesh, K., Abe, H., and Doi, Y. (2000) Synthesis, structure and properties of polyhydroxyalkanoates: biological polyesters, Progress in Polymer Science 25, 1503-1555.

3. Harding, K. G., Dennis, J. S., von Blottnitz, H., and Harrison, S. T. (2007) Environmental analysis of plastic production processes: comparing petroleum-based polypropylene and polyethylene with biologically-based poly-beta-hydroxybutyric acid using life cycle analysis, Journal of biotechnology 130, 57-66.

4. Philp, J. C., Bartsev, A., Ritchie, R. J., Baucher, M. A., and Guy, K. (2013) Bioplastics science from a policy vantage point, New biotechnology 30, 635-646.

5. Salehizadeh, H., and Van Loosdrecht, M. C. (2004) Production of polyhydroxyalkanoates by mixed culture: recent trends and biotechnological importance, Biotechnol Adv 22, 261-279.

6. Slater, S. C., Voige, W. H., and Dennis, D. E. (1988) Cloning and expression in Escherichia coli of the Alcaligenes eutrophus H16 poly-betahydroxybutyrate biosynthetic pathway, J Bacteriol 170, 4431-4436.

7. Hiroe, A., Tsuge, K., Nomura, C. T., Itaya, M., and Tsuge, T. (2012) Rearrangement of gene order in the phaCAB operon leads to effective production of ultrahigh-molecular-weight poly[(R)-3-hydroxybutyrate] in genetically engineered Escherichia coli, Applied and environmental microbiology 78, 3177-3184.

8. Yang, J. E., Choi, Y. J., Lee, S. J., Kang, K. H., Lee, H., Oh, Y. H., Lee, S. H., Park, S. J., and Lee, S. Y. (2013) Metabolic engineering of Escherichia coli for 
biosynthesis of poly(3-hydroxybutyrate-co-3-hydroxyvalerate) from glucose, Applied microbiology and biotechnology.

9. Heinrich, D., Madkour, M. H., Al-Ghamdi, M. A., Shabbaj, II, and Steinbuchel, A. (2012) Large scale extraction of poly(3-hydroxybutyrate) from Ralstonia eutropha H16 using sodium hypochlorite, AMB Express 2, 59. 